\title{
Potential Harmonics Expansion Method for Trapped Interacting Bosons : Inclusion of Two-Body Correlation
}

\author{
T. K. Das ${ }^{1}$, B. Chakrabarti ${ }^{2}$ \\ ${ }^{1}$ Department of Physics, University of Calcutta, 92 A. P. C. Road, Calcutta- 700009, \\ India. \\ e-mail:tkdas@cubmb.ernet.in,tkdas6@hotmail.com \\ ${ }^{2}$ Dept. of Physics and Astronomy, University of Oklahoma, Norman Ok 73019, U.S.A. \\ (present address: Department of Physics, K. N. College, Berhampore 742101, W.B., \\ India.) \\ e-mail:barnali@cubmb.ernet.in,barnali_chakrabarti@hotmail.com
}

\begin{abstract}
We study a system of $A$ identical interacting bosons trapped by an external field by solving ab initio the many-body Schrödinger equation. A complete solution by using, for example, the traditional hyperspherical harmonics $(\mathrm{HH})$ basis develops serious practical problems due to the large degeneracy of HH basis. Symmetrization of the wave function, calculation of the matrix elements, etc., become an immensely formidable task as $A$ increases. Instead of the HH basis, here we use a new basis, called "potential harmonics" (PH) basis, which is a subset of HH basis. We assume that the contribution to the orbital and grand orbital [in $3(A-1)$-dimensional space of the reduced motion] quantum numbers comes only from the interacting pair. This implies inclusion of two-body correlations only and disregard of all higher-body correlations. Such an assumption is ideally suited for the Bose-Einstein condensate (BEC), which is required - for experimental realization of BEC - to be extremely dilute. Hence three and higher-body collisions are almost totally absent. Unlike the $(3 A-4)$ hyperspherical variables in $\mathrm{HH}$ basis, the $\mathrm{PH}$ basis involves only three active variables, corresponding to three quantum numbers - the orbital $l$, azimuthal $m$, and the grand orbital $2 K+l$ quantum numbers for any arbitrary $A$. It drastically reduces the number of coupled equations and calculation of
\end{abstract}


the potential matrix becomes tremendously simplified, as it involves integrals over only three variables for any $A$. One can easily incorporate realistic atom-atom interactions in a straight forward manner. We study the ground and excited state properties of the condensate for both attractive and repulsive interactions for various particle number. The ground state properties are compared with those calculated from the Gross-Pitaevskii (GP) equation. We notice that our many-body results converge towards the mean field results as the particle number increases.

PACS number(s): 03.65.Ge, 03.75.Hh, 03.75.Nt, 31.15.Ja

Key words: Bose Einstein Condensation, Hyperspherical harmonics method, Potential harmonics. 


\section{Introduction}

Although the phenomenon of Bose Einstein Condensation (BEC) was known for a long time [1-3], its experimental observation in trapped and supercooled (down to nano Kelvin temperatures) alkali atoms in 1995 [4-6] renewed a great deal of interest - both experimental and theoretical - in the phenomemon. The importance of this topic is clearly demonstrated by the fact that two independent Nobel Prizes were awarded on BEC related works in quick succession in the recent past. The density of magneto-optically trapped atomic gas undergoing BEC is extremely low ( to avoid recombination of atoms through three and higher body collissions) and the number of trapped atoms is typically of the order of a few hundred to a few million. This is extremely small compared to the Avogadro number. For such a small number of atoms an exact $a b$ initio solution would have been ideally desirable. But an interacting system of $A=(N+1)$ particles has $3 N$ relative degrees of freedom and an ab initio solution of the corresponding Schrödinger equation is practically impossible for $A>3$. Hence the usual theoretical tools that have been used so far are the mean field models [7-10] and the Thomas-Fermi [8] approximation. The dilute atomic gas undergoes BEC below a critical temperature ( typically $10^{-9}$ degree $\mathrm{K}$ ) when most of the atoms (bosons) go to the single particle ground state. Then the de Broglie wavelength associated with the atomic motion is much larger than the interaction length scale. Hence the resulting many body system emerges as essentially a single quantum system where all the atoms behave in a coherent manner $[8,11]$. At zero temperature, the effect of the excited states are absent and the condensate is described by a single equation involving the condensate wave function [8]. However this simple picture is no more true at a finite temperature due to the existence of interparticle interactions. The usual procedure is to start with the mean field approximation like the Hartree-Fock (HF) theory for the many body system [7-10]. This is an independent particle approach where each individual atom is assumed to move in a single particle orbit. These orbits are determined self consistently by allowing an atom in one orbital to be influenced by other atoms in other orbitals through two-body interaction. Assuming a contact interaction for the two-body potential, viz., $V\left(\vec{r}-\overrightarrow{r^{\prime}}\right)=$ 
$g \delta\left(\vec{r}-\overrightarrow{r^{\prime}}\right)$, the many body equation reduces to the famous Gross-Pitaevskii (GP) equation [8]. At zero temperature, the effect of excited states are neglected and the condensate is described by the time independent GP equation

$$
\left[-\frac{\hbar^{2}}{2 m} \nabla^{2}+V_{\text {ext }}(\vec{r})+g \phi^{2}(\vec{r})\right] \phi(\vec{r})=\mu \phi(\vec{r})
$$

where $n(\vec{r})=\phi^{2}(\vec{r})$ is the condensate density and $\mu$ is the chemical potential. For a first approach the contact interaction is justified since in the cold and dilute gas only binary collissions at low energies are relevant. These are characterized by the $s$-wave scattering length $\left(a_{s c}\right)$, which is independent of the details of two-body potentials. The strength constant $g$ of the contact interaction is related to the scattering length through [8]

$$
g=\frac{4 \pi \hbar^{2} a_{s c}}{m}
$$

The GP equation has been used extensively to study the BEC [8,11]. Although most of the static, dynamic and thermodynamic properties are fairly well reproduced by the GP equation [8], the wave function does not include any correlation. Furthermore the assumption of a contact $\delta$-interaction is too simple and does not represent the realistic situation. It has already been shown that the Dirac $\delta$-function is not suitable as a replacement of the actual two-body interaction in exact theories in more than one dimension [12]. This is because the Hamiltonian then becomes unbound from below and the ground state energy diverges for an attractive zero range potential. Solutions are usually obtained in the metastable region (although such solutions are not rigorously correct for an attractive $\delta$-function potential) and the condensate becomes unstable for $N$ larger than a critical number, due to disappearance of the local minimum. This was shown by Bohn et al in a hyperspherical calculation keeping the lowest (most dominant) harmonic [13]. A third disadvantage is the non-lineraity of the GP equation, so that standard quantum mechanics is not applicable without concessional approximation. Thus one has to go beyond the mean field approximation and simple contact interactions.

Because of the limitations of the mean field theory and GP equation it is desirable to solve the many body linear Schrödinger equation directly. The Schrödinger equation for a system of $A=(N+1)$ identical bosons, each of mass $m$, confined by an 
external field $V_{\text {trap }}^{\prime}$ (acting on each individual boson) and interacting through a mutual two body interaction $V$ is

$$
\left[-\frac{\hbar^{2}}{2 m} \sum_{i=1}^{A} \nabla_{i}^{2}+\sum_{i=1}^{A} V_{\text {trap }}^{\prime}\left(\overrightarrow{x_{i}}\right)+\sum_{i<j=2}^{A} V\left(\overrightarrow{x_{i}}-\overrightarrow{x_{j}}\right)\right] \Psi(\vec{x})=E \Psi(\vec{x})
$$

where $\vec{x}$ refers to the set of particle coordinates $\left\{\overrightarrow{x_{1}}, \overrightarrow{x_{2}}, \ldots \ldots \overrightarrow{x_{A}}\right\}$ of $A$ bosons. The center of mass $(\mathrm{CM})$ motion can be eleminated resulting in a Schrödinger equation in $3 N$ variables. A standard practice is the use of hyperspherical harmonics expansion (HHE) method, in which the wave function is expanded in the complete set of hyperspherical harmonics $(\mathrm{HH})$ spanning the $(3 N-1)$-dimensional hyperangular space [14]. Projection on a particular $\mathrm{HH}$ leads to a system of coupled differential equations (CDE). However there are several very serious difficulties associated with the solution of a fairly large number of particles. Firstly the expansion basis of HH should be properly symmetrized and appropriate conserved quantum numbers properly taken care of. Secondly calculation of matrix elements of all the pairwise two-body potentials is an extremely formidable task. Finally, due to very large degeneracy of the HH basis for a large number of particles, the number of CDE and the dimemsion of the potential matrix is too large to be handled by any computer [14]. On top of all these, the convergence rate of the $\mathrm{HH}$ expansion, especially for long-range interactions, is slow [15]. For these reasons the HHE method has been used fully for the three body system only [15-18]. On the other hand, as we discussed earlier, the condensate can be treated broadly as a "single lump of quantum stuff", since all the individual atoms in the condensate lie within one single de Broglie wavelength [8]. Thus it is reasonable to assume that the basic properties of the condensate in the lowest approximation, is described by a single collective coordinate. This led Bohn et. al. [13] to go for the K-harmonic approximation, in which the HH expansion is restricted effectively to the first term only ( which is independent of the hyperangles). Such a drastic approximation may be justified for a contact interaction only. Even in this case, for an attractive $\delta$-function interaction, there are no rigorously stable solutions. Since the wave function becomes independent of the hyperangles and the hyperradius is invariant under any permutation of the particles, the wave function becomes totally symmetric, as required. The calculation of the potential matrix also simplifies immensely 
and the CDE reduces to a single differential equation [13]. The hyperradius emerges as the sought for collective coordinate. In spite of the great simplifications, there are serious criticisms of this approach : (1) The method cannot be applied to any realistic two-body interaction. (2) Even for a contact interaction, the method is not satisfactory for attractive $\delta$-function interaction, for which no rigorous solution extsits. (3) Only one collective variable is involved. Hence it can only describe the gross features of the condensate, without any finer details. Thus a more rigorous treatment is necessary. But as already mentioned a completely rigorous, essentially exact solution of the Schrödinger equation is possible for the three body system only. That has been done to get an idea of the initial trend as the particle number increases from three by Esry and Greene [12]. However that is far from the real situation in a condensate.

An alternative approach of exact numerical diagonalization of the many body Hamiltonian was adopted by Haugset and Haugerud [19] for a small number $(\leq 30)$ of interacting (via contact interaction) bosons confined by a harmonic trap. However, this was restricted to one and two dimensions only. Moreover the process is extremely time consuming even for two dimensional condensates, with a nagging question of convergence of the chosen harmonic oscillator basis expansion. The rate of convergence is expected to be slower for a realistic two-body interaction and in three dimensional condensates. Although analytic expressions for the matrix elements are greatly simplified for a delta function interaction, all the problems associated with a contact interaction discussed above remain for the two dimensional condensate. However, there is no problem with the one dimensional condensate, as one dimensional delta function is not pathological.

From the above discussion it is clear that an exact treatment of the many body system in three dimensions is not possible beyond the three body system. On the other hand the single quantum nature of the entire condensate suggests that out of the thousands to millions of degrees of freedom of the individual particles only a few are physically relevant. This is due to the fact that the condensate is possible only at extremely low temperatures ( low energy of the individual particles) and extremely low densities. Under these conditions only two body collisions are relevant. Three and higher body collsions are extremely rare and correlations beyond two body correlations in the 
condensate wave function are completely negligible upto a very high degree of precision. Indeed in an experimental situation this is ensured by keeping the density extremely low, so that there are no recombination via three and higher body collisions [8]. The mean field approach ignores all correlations including two-body correlations. Importance of two-body correlations in BEC has been emphasized by several authors [20,21]. Thus physically relevant quantities are contributed by two-body collisions, while the rest of the particles in the condensate do not partate in any motion other than a collective one and are simply inert spectators. The emerging picture then suggests that most of the degrees of freedom of these spectators can be frozen, while a single pair interacts. This reduces the physically important degrees of freedom of the condensate to just four - a global length scale (hyperradius) of the entire condensate, and the three degrees of freedom of the relative vector $\overrightarrow{r_{i j}}=\overrightarrow{x_{i}}-\overrightarrow{x_{j}}$ of the interacting pair. However one has to concede that any pair out of the $A=(N+1)$ atoms in the condensate can interact. These are also consistent with the intuitive "single quantum stuff" concept of the condensate.

Among the various possible theoretical approaches to handle the many body system, the HHE method appears to be the most lucrative one, as it readily provides the hyperradius as the most important collective variable. A theoretical formalism, arising out of the HHE method, was adopted by Fabre de la Ripelle [22] in 1986. Although the primary concern there was an application to the nuclear systems consisting of fermions, it was noted that the formalism is applicable to a system of identical bosons also [23]. To incorporate the importance of the interacting pair and two-body correlations, he introduced the potential harmonics $(\mathrm{PH})$ expansion basis [23], rather than the general $\mathrm{HH}$ basis, thereby reducing the expansion basis to a great extent. Potential harmonics is a subset of $\mathrm{HH}$, where all correlations higher than two-body ones are disregarded. In $\mathrm{PH}$, the contribution to the total orbital angular momentum as also the grand orbital quantum number comes only from the interacting pair. Here all the $(A-2)$ spectators are assumed to be described by the $\mathrm{HH}$ of the lowest (zero) order. We adopt this procedure since this approximation is quite justified in our situation due to the diluteness of BEC, where two-body correlation is the most important and all higher-body correlations can be safely ignored. Using Faddeev like decomposition of the total wave function, and then 
expanding each such component in an appropriate set of $\mathrm{PH}$, the number of CDE can be reduced drastically. Since the PH involves only four active degrees of freedom, calculation of potential matrix elements is simplified tremendously as compared to that in $\mathrm{HH}$ basis. Use of realistic two-body interactions and calculation of their matrix elements are quite straight forward. Requiring the Faddeev component for the $(i j)$ interacting pair to be symmetric under $(i j)$-pair exchange, the total wave function becomes automatically totally symmetric. Thus the symmetrization of the wave function is also handled properly.

Thus a truely many body equation is reduced to a tractable mathematical form. The assumptions leading to this are especially appropriate for the BEC. Hence we adopt the PH basis as our starting point. This is theoretically applicable to a system containing any number of particles, but we will see in Sec. III, that numerical difficulties arise as the number of particles increases beyond a certain number. In this communication we report some of the basic properties of the condensate for various particle numbers and compare them with previous calculations.

Sorensen et al [20,21] have followed a method which is similar in spirit to the present work, although it differs in details. They expand the wave function in the adiabatic subset $\Phi_{n}(\rho, \Omega)$ of the full $(N-1)$-body Hamiltonian (in CM frame). Later this is decomposed in Faddeev like components $\phi_{i j}$. This leads to an integro-differential equation (IDE) for $\phi\left(=\phi_{i j}\right.$, which is the same for all $i j$-pairs due to boson symmetry) involving five dimensional integrals and the full $(3 N-4)$-dimensional hyperangular differential operator $\hat{\Lambda^{2}}$. All $(3 N-5)$ angle derivatives other than $\alpha=\alpha_{12}$ (where $r_{i j}=\sqrt{2} \rho \sin \alpha_{i j}$ is the relative separation of the $(i j)$-pair and $\rho$ is the hyperradius of the full system), are disregarded, leaving only one angle variable. Assumption of a very short ranged two-body potential reduces the five dimensional integrals to two dimensional ones. In this limit simple expressions are obtained for the integrals in IDE. On the other hand, we write the complete $3 N$-dimensional Schödinger equation of the relative motion of a $(N+1)$ boson system in terms of Faddeev like components $\Phi\left(\overrightarrow{r_{i j}}, r\right)$, subject to the approximation that $\Phi\left(\overrightarrow{r_{i j}}, r\right)$ corresponds to zero eigenvalue of the hyper angular momentum operator (see later) for the $(N-1)$ remaining relative vectors of the specta- 
tors, while $(i j)$-pair interacts. These are then expanded in the potential harmonics $(\mathrm{PH})$ basis. The assumptions in our method are clearly justified in terms of the physics of the chosen system, which have been stated earlier. While the use of PH basis in nuclei (as originally used by Fabre in $[22,23])$ is questionable due to high spatial density of nucleons in a nucleus, its application in BEC is ideally suited (the number of atoms in the condensate is $\leq 10^{6}$ in a space of macroscopic linear dimensions of order $10^{-2} \mathrm{~cm}$, which is immensely smaller than the Avogadro number). As a consequence, the total orbital $(l)$ and grand orbital $(K)$ angular momenta of the system are contributed by the interacting pair alone. Apart from this well justified fundamental approximation, we need no other approximation. Although for the first calculation, we have restricted ourselves to $l=0$ and a central two-body interaction, both these can be relaxed resulting in a somewhat more complicated equation. Finally the system of coupled differential equations in one variable (hyperradius, $r$ ) can be solved numerically, without additional approximation (as done in ref. [15] and compared with adiabatic approximation (AA) in ref. [28]) using, e.g. renormalized Numerov method. Once again, as a preliminary calculation, we use AA to solve the CDE. Our use of AA in solving the CDE is not an indispensable one; it is done only to reduce the numerical calculation. But in the approach of Sorensen et $a l$, adiabatic subset is the starting point to separate the hyperangular and hyperradial motions. Furthermore our method can handle any two-body potential (central or not, short ranged or not); for non-central potential, calculation of matrix elements will involve integrals over two polar angles in addition. The approach of ref. [20,21] requries a very short ranged, central potential to reduce the equation to a manageable form. The present method has no such restriction.

The paper is organised as follows. In Sec. II, we present our choice of Jacobi coordinates and express the kinetic energy in the chosen set of hyperspherical variables. In the same section, we introduce the concept of potential harmonics basis and obtain the set of coupled differential equations resulting from the many-body Schrödinger equation. The numerical method for solving the CDE and results of our calculation are presented in Sec. III. There we compare our results for different numbers of particles with those of earlier calculations. Finally in Sec. IV we draw our conclusions. Some of 
the detailed expressions have been given in the Appendix.

\section{Theory}

\section{A. Choice of Jacobi coordinates}

We consider a system of $A=(N+1)$ identical bosons, each of mass $m$ and confined magnetically in a trap which is approximated by a spherically symmetric harmonic oscillator potential with frequency $\omega$. We assume that the atomic cloud is at zero temperature. The full many body Hamiltonian is given by

$$
\left[-\frac{\hbar^{2}}{2 m} \sum_{i=1}^{N+1} \nabla_{i}^{2}+\sum_{i=1}^{N+1} \frac{1}{2} m \omega^{2} x_{i}^{2}+\sum_{i j>i}^{N+1} V\left(\overrightarrow{x_{i}}-\overrightarrow{x_{j}}\right)\right] \Psi(\vec{x})=E^{\prime} \Psi(\vec{x})
$$

where $\vec{x}$ refers to the set of particle coordinates $\left\{\vec{x}_{1}, \vec{x}_{2}, \ldots, \vec{x}_{N+1}\right\}$ of $(N+1)$ bosons and $E^{\prime}$ is the total energy. We decompose the total wave function $\Psi(\vec{x})$ as the sum of pairwise partial waves

$$
\Psi(\vec{x})=\sum_{i j>i}^{N+1} \psi_{i j}(\vec{x})
$$

The Schrödinger equation for $\psi_{i j}$ can be written as

$$
\left(T+V_{\text {trap }}^{\prime}-E^{\prime}\right) \psi_{i j}(\vec{x})=-V\left(r_{i j}\right) \sum_{k l>k}^{N+1} \psi_{k l}(\vec{x})
$$

where $T$ is the total kinetic energy operator, $V_{\text {trap }}^{\prime}$ is the confining potential, $V_{\text {trap }}^{\prime}=$ $\sum_{i=1}^{N+1} \frac{1}{2} m \omega^{2} x_{i}^{2}$ and $V\left(r_{i j}\right)$ is the pairwise local central two-body interaction between $i^{t h}$ and $j^{\text {th }}$ particles, $\overrightarrow{r_{i j}}=\overrightarrow{x_{i}}-\overrightarrow{x_{j}}$. Applying the operator $\sum_{i j>i}^{N+1}$ on both sides of eq.(6), and using eq.(5), we get back eq.(4). Now instead of $(N+1)$ particle coordinates $\overrightarrow{x_{i}}$, the system can alternatively be described by the center of mass coordinate $\vec{R}$

$$
\vec{R}=\frac{1}{N+1} \sum_{i=1}^{N+1} \overrightarrow{x_{i}}
$$

and $N$ Jacobi coordinates defined as

$$
\vec{\zeta}_{i}=\sqrt{\frac{2 i}{i+1}}\left(x_{i+1}-\frac{1}{i} \sum_{j=1}^{i} \overrightarrow{x_{j}}\right), \quad i=1, \ldots, N .
$$


The chosen normalization of $\vec{\zeta}_{i}$ facilitates writing the Laplace operator in the form

$$
\frac{1}{2} \sum_{i=1}^{N+1} \nabla_{i}^{2}=\frac{1}{2 A} \nabla_{R}^{2}+\sum_{i=1}^{N} \nabla_{\zeta_{i}}^{2}
$$

Then the relative motion (after removal of center of mass motion from eq.(4)) is described by $[14,23]$

$$
\left[-\frac{\hbar^{2}}{m} \sum_{i=1}^{N} \nabla_{\zeta_{i}}^{2}+V_{\text {trap }}+V_{\text {int }}\left(\overrightarrow{\zeta_{1}}, \ldots, \overrightarrow{\zeta_{N}}\right)-E\right] \psi\left(\overrightarrow{\zeta_{1}}, \ldots, \overrightarrow{\zeta_{N}}\right)=0
$$

where $V_{\text {trap }}=\sum_{i=1}^{N} \frac{1}{2} m \omega^{2} \zeta_{i}^{2}$ and $V_{\text {int }}$ is the sum of all pairwise interactions, $V_{\text {int }}=$ $\sum_{i j>i}^{N+1} V\left(r_{i j}\right)$ expressed in the relative coordinates. Here $E$ is the energy of the relative motion, i.e., $E^{\prime}$ minus energy of CM motion. The hyperradius $r$ is defined as [22]

$$
r=\left[\sum_{i=1}^{N} \zeta_{i}^{2}\right]^{1 / 2}=\left[\frac{2}{N+1} \sum_{i, j>i} r_{i j}^{2}\right]^{1 / 2}
$$

which is invariant under permutations of the particle indices as also three dimensional rotations. The hyperspherical coordinates are constituted by the hyperradius $r$ and remaining $(3 N-1)$ hyperangles, denoted collectivelty by $\Omega_{N}$ in $D=3 N$ dimensional space. Note that the choice of Jacobi coordinates eq.(8), is not unique, since the labelling of the particle indices and consequently that of the Jacobi coordinates are arbitrary. We choose a particular set by specifying the relative separation of the interacting pair, $\overrightarrow{r_{i j}}$ as $\overrightarrow{\zeta_{N}}$ and $(\vartheta, \varphi)$ are the two spherical polar coordinates associated with $\overrightarrow{r_{i j}}$. The relative length is defined in terms of $\phi$ through $r_{i j}=r \cos \phi$. For the rest of $(N-1)$ Jacobi coordinates, we define the hyperradius $\rho_{i j}$ in the $3(N-1)$ dimensional space by

$$
\rho_{i j}=\left[\sum_{k=1}^{N-1} \zeta_{k}^{2}\right]^{1 / 2}
$$

which is related with $\overrightarrow{\zeta_{N}}=\overrightarrow{r_{i j}}$ by

$$
\rho_{i j}^{2}+r_{i j}^{2}=r^{2}, \quad \rho_{i j}=r \sin \phi \quad .
$$

Then our hyperspherical coordinates become

$$
\left(r, \Omega_{N}\right)=\left(r, \phi, \vartheta, \varphi, \Omega_{N-1}\right) \quad
$$


Here $\Omega_{N-1}$ involves $2(N-1)$ spherical polar angles associated with each of $(N-1)$ Jacobi vectors $\left\{\vec{\zeta}_{1}, \vec{\zeta}_{2}, \ldots, \vec{\zeta}_{N-1}\right\}$ and $(N-2)$ angles (expressing relative lengths), i.e., a total of $(3 N-4)$ variables. In this choice of hyperspherical coordinates, the Laplace operator takes the form $[22]$

$$
\nabla^{2} \equiv \sum_{i=1}^{N} \nabla_{\zeta_{i}}^{2}=\frac{\partial^{2}}{\partial r^{2}}+\frac{3 A-4}{r} \frac{\partial}{\partial r}+\frac{L^{2}\left(\Omega_{N}\right)}{r^{2}}, \quad A=N+1
$$

$L^{2}\left(\Omega_{N}\right)$ is the grand orbital operator in $3 N$ dimensional space which is obtained from a recurrence formula [22] and has the form

$$
L^{2}\left(\Omega_{N}\right)=4\left(1-z^{2}\right) \frac{\partial^{2}}{\partial z^{2}}+6[2-N(1+z)] \frac{\partial}{\partial z}+2 \frac{l^{2}\left(\omega_{i j}\right)}{1+z}+2 \frac{L^{2}\left(\Omega_{N-1}\right)}{1-z}
$$

where $z=\cos 2 \phi, \omega_{i j}$ reprsents the two polar angles $(\vartheta, \varphi)$ associated with $\overrightarrow{r_{i j}}$ and $L^{2}\left(\Omega_{N-1}\right)$ is the grand orbital operator in $3(N-1)$ dimensional space.

\section{B. Potential basis and potential multipoles}

To exapand a function $V\left(r_{i j}\right)$ in hyperspherical harmonics $(\mathrm{HH})$ we use the above definition of Jacobi coordinates. It is easy to see that HH basis which is complete for the expansion of $V\left(r_{i j}\right)$ does not contain any function of the coordinate $\vec{\zeta}_{i}$ with $i<N$ and is given by [23]

$$
\mathcal{P}_{2 K+l}^{l, m}\left(\Omega_{i j}\right)=Y_{l}^{m}\left(\omega_{i j}\right)^{(N)} P_{2 K+l}^{l, 0}(\phi) \mathcal{Y}_{0}(D-3)
$$

where ${ }^{(N)} P_{2 K+l}^{l, 0}$ is a function involving the Jacobi polynomial and is needed in the general expression of the hyperspherical harmonics (see Appendix) of grand orbital $2 K+l$ and orbital angular momemtum $l$. The quantity $\mathcal{Y}_{0}(D-3)$ is the $\mathrm{HH}$ of order zero (i.e. grand orbital quantum number is zero) in $3(N-1)$ dimensional space, $\mathcal{Y}_{0}(D-3)=$ $\left(\frac{\Gamma((D-3) / 2)}{2 \pi^{(D-3) / 2}}\right)^{1 / 2}$. This new basis set which is a subset constituted by HH of order $(2 K+l)$ are called "potential harmonics" $(\mathrm{PH})$. These are the eigenfunctions of $L^{2}\left(\Omega_{N}\right)$, when the eigenvalue of $L^{2}\left(\Omega_{N-1}\right)$ is 0 and satisfy the eigenvalue equation :

$$
\left[L^{2}\left(\Omega_{N}\right)+\mathcal{L}(\mathcal{L}+D-2)\right] \mathcal{P}_{2 K+l}^{l, m}\left(\Omega_{i j}\right)=0, \quad \mathcal{L}=2 K+l
$$

The relation $L^{2}\left(\Omega_{N-1}\right) \psi_{i j}(\vec{x})=0$ implies that we are considering only those states which are invariant under all generalized rotations in $3(N-1)$ dimensional space. Natuarally 
the contribution to the grand orbital quantum number comes only from the interacting pair. This corresponds effectively to two-body correlations only in the wave function. Due to diluteness of atomic BEC, the effect of higher body correlations can be ignored as the probability for three or more particles to come close at the same time is extremely small. This reduces the number of quantum numbers in the new basis (all the quantum numbers specifying the eigenfunctions of $L^{2}\left(\Omega_{N-1}\right)$ are zero). It contains only three quantum numbers; orbital $l$, azimutal $m$ and grand orbital $2 K+l$ for any $N$, instead of $(3 N-1)$ quantum numbers corresponding to $(3 N-1)$ hyperspherical variables in the general HH basis. The normalization condition is given by

$$
\int \mathcal{P}_{2 K+l}^{l, m^{*}}\left(\Omega_{i j}\right) \mathcal{P}_{2 K^{\prime}+l^{\prime}}^{l^{\prime} m^{\prime}}\left(\Omega_{i j}\right) d \Omega_{i j}=\delta_{K K^{\prime}} \delta_{l l^{\prime}} \delta_{m m^{\prime}}
$$

Then the PH expansion of the potential is

$$
V\left(r_{i j}\right)=\sum_{K, l, m} A_{l}^{m}(i, j) \mathcal{P}_{2 K+l}^{l, m}\left(\Omega_{i j}\right) V_{K}^{(D, l)}(r)
$$

$A_{l}^{m}(i, j)$ is an operator which is independent of $r_{i j}$, but may act on other variables like

spin variables. The quantity $V_{K}^{(D, l)}(r)$ are the "potential multipoles" and for a central potential, it is given by [23]

$$
\begin{aligned}
V_{K}^{(D, l)}(r) & =\left\langle\mathcal{P}_{2 K+l}^{l, m}\left(\Omega_{i j}\right)\right| V\left(r_{i j}\right)> \\
& =\left|\mathcal{Y}_{0}(D-3)\right|^{-1} \int_{0}^{\pi / 2}(N) P_{2 K+l}^{l, 0}(\phi) V_{l}(r \cos \phi)(\sin \phi)^{D-4}(\cos \phi)^{2} d \phi
\end{aligned}
$$

where the functions ${ }^{(N)} P_{2 K+l}^{l, 0}(\phi)$ are defined in the Appendix. Starting from the multipoles calculated either for the $D=5$ or $D=6$ ( depending wheather $D$ is odd or even) and using simple recurrence formulæ potential multipoles for any D can be calculated $[23]$.

\section{Coupled differential equations}

Splitting eq.(10) in the manner of eq.(6) for the $(i j)$-interacting pair and using eqs.(14)(16), subject to the restriction that the eigenvalue of $L^{2}\left(\Omega_{N-1}\right)$ is zero, we see that the (ij) Faddeev component will be a function of $\vec{r}_{i j}$ and $r$ only and satisfies [23]

$$
\left(T+V_{\text {trap }}-E\right) \Phi\left(\overrightarrow{r_{i j}}, r\right)=-V\left(r_{i j}\right) \sum_{k, l>k} \Phi\left(\overrightarrow{r_{k l}}, r\right)
$$


where $\Phi\left(\overrightarrow{r_{i j}}, r\right)$ differs from the general solution $\psi_{i j}$ by the fact that it corresponds to eigenvalue zero of the operator $L^{2}\left(\Omega_{N-1}\right)$. Next expand the wave function $\Phi\left(\overrightarrow{r_{i j}}, r\right)$ in the complete set of potential harmonics (when $l$ is a good quantum number) as

$$
\Phi\left(\overrightarrow{r_{i j}}, r\right)=r^{-\frac{D-1}{2}} \sum_{K^{\prime}} \mathcal{P}_{2 K^{\prime}+l}^{l m}\left(\Omega_{i j}\right) u_{K^{\prime}}^{l}(r)
$$

Substitution of eq.(23) in eq.(22) and projection on the same basis, leads to the set of coupled differential equations [23]

$$
\left[-\frac{\hbar^{2}}{m} \frac{d^{2}}{d r^{2}}+\frac{\hbar^{2}}{m} \frac{\mathcal{L}_{K}\left(\mathcal{L}_{K}+1\right)}{r^{2}}+V_{t r a p}(r)-E\right] u_{K}^{l}(r)+\sum_{K^{\prime}} f_{K^{\prime} l}^{2} V_{K K^{\prime}}(r) u_{K^{\prime}}^{l}(r)=0
$$

where

$$
\begin{aligned}
\mathcal{L}_{K} & =2 K+l+\frac{D-3}{2} \\
f_{K l}^{2} & =\sum_{k, l>k}<\mathcal{P}_{2 K+l}^{l m}\left(\Omega_{i j}\right) \mid \mathcal{P}_{2 K+l}^{l m}\left(\Omega_{k l}\right)>
\end{aligned}
$$

The potential matrix is given by

$$
V_{K K^{\prime}}(r)=\int \mathcal{P}_{2 K+l}^{l m^{*}}\left(\Omega_{i j}\right) V\left(r_{i j}\right) \mathcal{P}_{2 K^{\prime}+l}^{l m}\left(\Omega_{i j}\right) d \Omega_{N}
$$

So instead of $(3 N-1)$ angle variables in HHE method, in potential harmonics expansion method (PHEM) the integral invloves only 3 angle variables. It greatly simplifies the calculation of the matrix element for any $N$.

The quantity $f_{k l}^{2}$ of eqs. (24) and (25) is given by [23]

$$
f_{K l}^{2}=1+\left[2(A-2)\left(-\frac{1}{2}\right)^{l} P_{K}^{\alpha \beta}\left(-\frac{1}{2}\right)+\frac{(A-2)(A-3)}{2} P_{K}^{\alpha \beta}(-1) \delta_{l, 0}\right] / P_{K}^{\alpha \beta}(1)
$$

where $\alpha=(3 A-8) / 2$ and $\beta=l+\frac{1}{2}$ and $P_{K}^{\alpha \beta}(x)$ is the Jacobi polynomial. Multiplying eq. (24) by appropriate constant factors, it can be put in a symmetric form:

$$
\begin{aligned}
& {\left[-\frac{\hbar^{2}}{m} \frac{d^{2}}{d r^{2}}+\frac{\hbar^{2}}{m r^{2}}\{\mathcal{L}(\mathcal{L}+1)+4 K(K+\alpha+\beta+1)\}+V_{\text {trap }}(r)-E\right] U_{K l}(r) } \\
+ & \sum_{K^{\prime}} \bar{V}_{K K^{\prime}}(r) U_{K^{\prime} l}(r)=0
\end{aligned}
$$

where $\mathcal{L}=l+(3 A-6) / 2$, the symmetrized potential matrix $\bar{V}_{K K^{\prime}}$ has the form

$$
\bar{V}_{K K^{\prime}}(r)=f_{K l} V_{K K^{\prime}}(r) f_{K^{\prime} l}\left(h_{K}^{\alpha \beta} h_{K^{\prime}}^{\alpha \beta}\right)^{-\frac{1}{2}}
$$

and

$$
U_{K l}(r)=f_{K l}\left(h_{K}^{\alpha \beta}\right)^{\frac{1}{2}} u_{K}^{l}(r)
$$


Here $h_{K}^{\alpha \beta}$ is the norm of the Jacobi polynomial $P_{K}^{\alpha \beta}(x)$ [24]. The potential matrix element is obtained from eq. (26), using eq. (17) and eq. (42) of Appendix, in the form

$$
V_{K K^{\prime}}(r)=\int_{-1}^{+1} P_{K}^{\alpha \beta}(z) V\left(r \sqrt{\frac{1+z}{2}}\right) P_{K^{\prime}}^{\alpha \beta}(z) w_{l}(z) d z,
$$

where $w_{l}(z)=(1-z)^{\alpha}(1+z)^{\beta}$ is the weight function of the Jacobi polynomials [24]. For Gaussian interaction with $A=3$, the integral can be obtained analytically [25], from where one can directly check the numerical accuracy.

\section{Numerical method and results}

\section{A. Numerical method}

For a chosen number of particles $(A)$ and a chosen interaction potential $\left(V\left(r_{i j}\right)\right)$, we calculate the potential matrix for a fixed value of hyperradius $(r)$ from eqs. (29) and (31) using a multi-point Gauss-Jacobi quadrature. For the present calculation we select $l=0$ and truncate the $\mathrm{PH}$ expansion basis of eq.(23) to a maximum $K$ value $\left(=K_{\max }\right)$. In order to simplify the solution of the set of coupled differential equations, eq. (28), we adopt the hyperspherical adiabatic approximation (HAA) $[16,26]$. In this approximation it is assumed that the hyperradial motion is slow compared to the hyperangular motions. Hence the latter can be solved adiabatically for a fixed value of $r$ to get an effective potential as a parametric function of $r$ [16]. This is done by diagonalizing the potential matrix together with the diagonal hypercentrifugal repulsion and the trapping potential for each value of $r$ :

$$
\sum_{K^{\prime}=1}^{K_{\max }} M_{K K^{\prime}}(r) \chi_{K^{\prime} \lambda}(r)=\omega_{\lambda}(r) \chi_{K \lambda}(r)
$$

where

$$
M_{K K^{\prime}}(r)=\bar{V}_{K K^{\prime}}(r)+\left[\frac{\hbar^{2}}{m r^{2}}\{\mathcal{L}(\mathcal{L}+1)+4 K(K+\alpha+\beta+1)\}+V_{\text {trap }}(r)\right] \delta_{K K^{\prime}}
$$

The lowest eigenvalue gives the "lowest eigen potential ", $\omega_{0}(r)$. As we discussed in the introduction, the hyperradius behaves as the most important collective coordinate and $\omega_{0}(r)$ is the potential in which the condensate moves as a "single quantum stuff", 
except for attractive two-body interactions and $A>A_{c r}$ (see later). Another collective coordinate is the hyperangle $\phi$ appearing in the wavefunction through eqs. (23) and (17), which describes the deviations of the condensate from hyperspherically symmetric distribution.

In the HAA approach, an approximate solution of eq. (28) is obtained by solving a single uncoupled differential equation [16]

$$
\left[-\frac{\hbar^{2}}{m} \frac{d^{2}}{d r^{2}}+\omega_{0}(r)+\sum_{K=0}^{K_{\max }}\left|\frac{d \chi_{K 0}(r)}{d r}\right|^{2}-E\right] \zeta_{0}(r)=0
$$

The solution of eq. (34) subject to appropriate boundary conditions on $\zeta_{0}(r)$ gives the energy $E$, which is an upper bound for the eigen energy of eq. (28). The partial waves of eq.(28) are given in HAA by [16]

$$
U_{K l}(r) \simeq \zeta_{0}(r) \chi_{K 0}(r)
$$

This approximation is usually called uncoupled adiabatic approximation (UAA) in the literature $[16,26]$; disregarding the third term on the left side of eq.(34) one gets the so called extreme adiabatic approximation (EAA). It has been shown that the HAA is in very good agreement (having less than $1 \%$ error) with the exact solution of the CDE for both atomic [27-29] and nuclear [30-31] cases. Since this is adequate for this preliminary application of this new method, we adopt the HAA, instead of solving the full set of CDE by exact numerical algorithm like the renormalized Numerov method [32].

\section{B. Choice of two body interaction potential}

In this report we compare our results with those of the GP equation as also with other calculations using a contact $\delta$-interaction. But a $\delta$-function interaction is not a physical one since it diverges at $r_{i j}=0$ and nothing (e.g. centrifugal repulsion) can prevent its overwhelming effect. As a result, the Hamiltonian becomes unbound from below for an attractive $\delta$ interaction. This is manifest in the effective potential $\omega_{0}(r)$, which for a particle number $(A)$ less than a critical value $\left(A_{c r}\right)$ produces a local minimum at a finite value of $r$ ( giving rise to a metastable solution), but $\omega_{0}(r) \rightarrow-\infty$ as $r \rightarrow 0$ for any number of particles (see following subsection, as also ref. [12]). Thus 
there are no rigorously acceptable and stable solution for any $A$, since the attractive essential singularity at $r=0$ will pull the system to $r \rightarrow 0$ and the corresponding wave function will diverge at $r=0$. Although the $\delta$-function is particularly convenient for analytic calculations, it is desirable to choose an interaction which would either remain finite or at worst introduce a removable singularity as $r_{i j} \rightarrow 0$ for attractive cases. Then the hyper centrifugal repulsion in eq.(28) (which is non vanishing even for $l=0, K=0$ and increases rapidly as $A$ increases) will not allow the interacting particles to come too close to each other. We thus choose a Gaussian potential of strength $V_{0}$ and range $r_{0}$

$$
V\left(r_{i j}\right)=V_{0} e^{-\frac{r_{i j}^{2}}{r_{0}^{2}}}
$$

Choosing appropriate values of $V_{0}$ and $r_{0}$, the potential can be made either soft or stiff. A particular experimental situation at the low temperature limit is characterized by the $s$-wave scattering length $\left(a_{s c}\right)$. For given values of $V_{0}$ and $r_{0}$, one can calculate $a_{s c}$ by solving the two-body radial Schrödinger equation for positive energies, in the zero energy limit. Alternately, for a suitably chosen value of $r_{0}$ and an experimentally known value of $a_{s c}$ one can find $V_{0}$ numerically from the solution of the two-body Schrödinger equation in the $E \rightarrow 0+$ limit. In Fig. 1, we present a plot of calculated $a_{s c}$ as a function of $V_{0}$ for $r_{0}=0.0855$ o.u.. As is well known, $a_{s c}$ is positive and monotonically continuous for $V_{0}>0$. The scattering length becomes negative as $V_{0}$ becomes negative and continues to $-\infty$ at a particular negative value of $V_{0}$. At this point, $a_{s c}$ has an infinite discontinuity and as $V_{0}$ decreases further, $a_{s c}$ starts from $+\infty$ and decreases continuously to $-\infty$ at a second particular value of $V_{0}$. The first, second, ..., branch of the curve (as $V_{0}$ decreases from positive values) correspond respectively to zero, one, ..., two-body bound states. For a stable BEC, we choose the first branch of the curve. From Fig. 1, one notices that for $r_{0}=0.0855$ o.u., the first discontinuity occurs at about $V_{0}=-184$ o.u. For $r_{0}=$ 0.005 o.u., this value is much more negative $\left(-8.18963 \times 10^{5}\right.$ o.u. $)$. In the same figure, we also plot the Born approximation for $a_{s c}$ (corresponding to $r_{0}=0.0855$ o.u.), given by $[12]$

$$
a_{s c}^{(B)}=\frac{m}{2 \pi \hbar^{2}} \int d^{3} r V(\vec{r})
$$


For a Gaussian interaction this integral can be done analytically and gives

$$
a_{s c}^{(B)}=\frac{2 m}{\hbar^{2}} V_{0} r_{0}^{3} \frac{\sqrt{\pi}}{4}
$$

From Fig. 1, it is seen that the Born approximation is good only for small values of $\left|V_{0}\right|$. In this work, we use the exact result and not the Born approximation. For repulsive potentials, we choose a conveniently small value of $r_{0}$ and calculate $V_{0}$ by the exact procedure.

Choosing a smaller value of $r_{0}, V_{0}$ increases in magnitude and the potential becomes stiffer. For very small values of $r_{0}, V\left(r_{i j}\right)$ simulates a $\delta$-function. For attractive interactions, we perform a model calculation with chosen values of $r_{0}$ and $V_{0}$.

\section{Results}

With this choice of potential we have solved the CDE eq.(28) for various number of particles. We use oscillator units (o.u.) in which energy and length are ex-

pressed in units of oscillator energy and oscillator length $\left(\hbar \omega\right.$ and $\sqrt{\frac{\hbar}{m \omega}}$ respectively, where $\omega$ is the circular frequency of the harmonic confining potential). The matrix element, eq. (31), has been calculated by a multi-point Gauss-Jacobi quadrature, the number of points being decided by the condition of convergence of a typical matrix element. We first verify that our results are independent of the choice of $r_{0}$, if $V_{0}$ is appropriately calculated using two-body Schrödinger equation, so that $a_{s c}$ has the same value ( 100 Bohr for ${ }^{87} R b$, which has a repulsive interaction). In a few representative calculations, the ground state energy and low lying excitation spectrum of the condensate containing $A$ particles have been found to be stable within numerical errors, for several values of $r_{0}$ ranging from 0.1 o.u. to 0.005 o.u. As for example, the ground state energy per particle for a condensate containing $A=10$ bosons approaches a convergence as $r_{0}$ decreases from 0.1 to 0.005 . Relative change in the energy per particle from $r_{0}=0.01$ o.u. to 0.005 $o . u$. is only about $0.012 \%$. As $r_{0}$ decreases, the calculation of the matrix elements as also the solution of eq. (34) become extremely CPU time consuming. This is because for very small $r_{0}$, one has to introduce very fine $r$-mesh intervals (typically $10^{-5}$ o.u.), which increases CPU time enormously. To keep the numerical calculations manageable, 
we choose $r_{0}=0.005 o . u$. and $V_{0}=3.1985 \times 10^{6}$ o.u. (which corresponds to JILA ${ }^{87} R b$ experiments with $a_{s c}=100$ Bohr and trap frequency $\nu=200 \mathrm{~Hz}$ ). We next test the convergence of our results as $K_{\max }$ increases by calculating the ground state energy per particle of the condensate for $V_{0}=3.1985 \times 10^{6}$ o.u. and $r_{0}=0.005$ o.u. Our results are presented in Table 1. It is seen that the energy per particle converges quite rapidly as $K_{\max }$ increases. For example, for $A=20$, the change in energy is less than $0.001 \%$ as $K_{\max }$ increases from 2 to 10 . Another interesting observation is that the ground state energy decreases as $K_{\max }$ increases, which is consistent with the Rayleigh-Ritz principle. Thus it is reassuring that our method is working satisfactorily and is fast converging.

However a numerical difficulty appears as the particle number $(A)$ and $K_{\max }$ increase. The quantity $\alpha$ increases rapidly with $A$, (e.g., $\alpha=0.5$ for $A=3$ and $\alpha$ $=71$ for $A=50$ ), while $\beta$ remains constant at $\frac{1}{2}$ (for $l=0$ ). Thus the Jacobi polynomial $\left(P_{n}^{\alpha, \beta}(z)\right)$ as also its weight function $\left(w_{l}(z)\right)$ are highly asymmetric functions in the interval $[-1,1]$ (see ref. [24]). They have tremendous variation in their values (e.g. $2^{\alpha}$ to zero) as the argument varies from -1 to +1 for large $A$. Furthermore $w_{l}(z)$ increases from 0 to $2^{\alpha}$ within a very small interval close to $z=-1$, for large $\alpha$. In addition, $P_{n}^{\alpha, \beta}(z)$ has $n$ nodes in the interval $[-1,1]$. Hence unavoidable numerical error creeps into the numerical integration of the potential matrix, using eq. (31). Consequently the calculated energy per particle and other physical quantities show irregularity for $A \geq 40$, as also for smaller $A$ with large $K_{\max }$. Therefore we have restricted $A$ to 35 . Even for $15 \leq A \leq 35$, some results for large $K_{\max }$ are not reliable. Hence these have been left out in Table 1. In all subsequent calculations, we keep $K_{\max }=4$. We are at present trying to overcome these difficulties for large $A$ by improved numerical techniques.

In Fig. 2, we present a plot of the lowest eigen potential in EAA for $A$ $=20$, for a model replusive interaction with $V_{0}=20$ o.u. and $r_{0}=0.1$ o.u. (dotted curve) corresponding to $a_{s c}=0.01553$ o.u. (224.3 Bohr). In the same figure, we also include the non-interacting $\left(V_{0}=0, a_{s c}=0\right)$ case (continuous curve), which naturally lies below the repulsive interaction $\left(a_{s c}>0\right)$ curve. In Fig. 3, we plot $\omega_{0}(r)$ for an attractive interaction, viz., $V_{0}=-100$ o.u., $r_{0}=0.0855$ o.u (note from Fig. 1 that this corresponds to zero two-body bound state and $a_{s c}=-0.1176$ o.u.) for $A=10$. Since we cannot 
go to large values of $A$ due to numerical problems mentioned above, we keep $A$ small and increase $V_{0}$ to study the critical behaviour (see below) at a lower value of $A$. Both these curves have the general features same as those found in earlier calculations using K-harmonics approximation [13]. Fig. 3 shows a metastable region with a local minimum of $\omega_{0}(r)$, which is preceded by a collapse region for smaller $r$. As $A$ increases above a critical value $\left(A_{c r}\right)$, the metastable region disappears. This is seen in Fig. 4 for $A=16$ for the same $V_{0}$ and $r_{0}$. These features are the same as reported earlier [13]. However, in our case, since $V(r)$ is finite for $r \rightarrow 0$, and the repulsive centrifugal term goes as $\frac{1}{r^{2}}$, there is no real collapse. For very small $r, \omega_{0}(r)$ becomes strongly repulsive even for an attractive two body interaction. This is represented by the dotted lines in Figs. 3 and 4. Note that the dotted and continuous parts together constitute the entire calculated $\omega_{0}(r)$ curve. The small $r$ (repulsive) part is plotted with a different (dotted) curve to emphasize that the remaining part (continuous portion) of $\omega_{0}(r)$ has the same behaviour as obtained with attractive contact interaction in ref. [13]. Only the dotted part differs remarkably from the corresponding part in ref. [13]. In reality for $A>A_{c r}$, there is a very narrow and deep well at a small value of $r$; hence all the particles will be trapped within this well. As the particles come within a small region, corresponding to a small value of $r$, the density of the condensate increases, and due to increased three and higher body collisions, molecule formation takes place with the disappearance of the BEC. The deep and narrow well in $\omega_{0}(r)$ near the origin, for an attractive two-body interaction with $A>A_{c r}$, can support a lowlying, highly localized bound state, which describes the formation of molecules. Although this is the lowest lying state in the corresponding $\omega_{0}(r)$, it does not represent the ground state of the condensate, which has already "collapsed". This gives a realistic scenario of what happens as $A$ increases above $A_{c r}$ for attractive interactions. For an attractive $\delta$-function interaction, the lack of a rigorous solution fails to give a realistic picture and one talks of a "collapse of the condensate" in a qualitative fashion.

We next calculate first three excited states for different number of particles $(A)$ in the condensate. These are shown in Fig. 5. Values of $E_{n}^{e x}$ for $n=1,2,3$ have been represented by diamonds, pluses and squares respectively. The excitation energy 
increases slowly with $A$. They agree fairly well with the K-harmonic approximation [13].

In Table 2, we present numerical values and notice that the excitation energies increase gradually with $A$.

In Fig. 6, we plot the ground state wave function, $\zeta_{0}(r)$, as a function of the global length $r$ for various values of $A$. It is seen that as the particle number increases, the peak of $\zeta_{0}(r)$ shifts towards larger values of $r$. This is understandable, since for large $A$, the total repulsion of all the pairs increases as $A^{2}$ and particles are pushed outwards, by the $A$-dependent hypercentrifugal repulsion in eq. (28).

Finally we calculate and plot the ground state energy per particle $\left(E_{0} / A-\frac{3}{2} \hbar \omega\right)$ as a function of $A a_{s c}$ for selected values of $A(10,20$ and 30$)$ for a repulsive interaction in Fig. 7. Corresponding curves are from the bottom upwards respectively. We compare these with the corresponding values calculated from the GP equation. This curve is the top most in Fig. 7. One notices that our results approach the GP result as $A$ increases for a fixed $A a_{s c}$, as expected. We also note that our energies are below those of the GP equation, indicating once again a better result from the variational point of view. Fig. 7 agrees qualitatively with a similar figure of ref. [19], where exact diagonalization of the Hamiltonian was performed for one and two dimensional condensates respectively.

\section{Conclusions}

In this communication, we have investigated the $T=0$ properties of a Bose-Einstein condensate (BEC), consisting of $A$ atoms (bosons) trapped by an external field and interacting via realistic two-body interactions. An ab initio treatment of the Schrödinger equation involves $3(A-1)$ degrees of freedom for the relative motion. Use of traditional hyperspherical harmonics expansion (HHE) method is impossible for $A>3$, due to tremendous and mounting complexity of the method as particle number increases beyond three. We circumvent this difficulty by exploiting the subset of potential harmonics (PM) basis, instead of the full set of hyperspherical harmonics ( $\mathrm{HH})$ basis. The $\mathrm{PH}$ basis is obtained as the subset of $\mathrm{HH}$ needed for expanding the two-body potential for the interacting pair. The choice of $\mathrm{PH}$ basis corresponds to inclusion of two-body 
correlations and disregard of all higher-body correlations in the condensate. On the other hand, two-body correlations are very important in BEC and cannot be disregarded as in mean field theories or the GP equation. This assumption is exactly appropriate for the BEC, since for practical realization of BEC, the density of atoms must be kept so low that there are practically no three and higher body collisions. Existence of the latter type of collisions would facilitate formation of molecules and consequent depletion of the condensate. As a consequence of this assumption, only four active degrees of freedom of the condensate (instead of a total of $3 A-3$ degrees of freedom for the relative motion of the $A$ particle system) are physically important - these are constituted by the global length (hyperradius, $r$ ) and the three active angle variables of the $\mathrm{PH}$. In effect one freezes the remaining $(3 A-7)$ angle variables of $\mathrm{PH}$. This leads to a tremendous simplification of the actual numerical calculation. Since we make Faddeev like decomposition of the full wave function, an appropriate symmetrization of the wave function under exchange of the interacting pair guarantees full symmetrization. Moreover, the potential matrix elements involve integrals over only three angle variables, leading to an immense reduction in the complexity of the numerical procedure for $A$. Since there are no theoretical restrictions on $A$, this opens the possibility of an approximate but very reliable, ab initio solution of the large but finite body condensate. However, a numerical difficulty arises due to the fact that the parameter $\alpha(=(3 A-8) / 2)$ of the Jacobi polynomials, $P_{n}^{\alpha, \beta}(x)$, and its associated weight function, become very large as $A$ increases. These cause numerical problems, for $A \geq 40$. We are at present attempting to remove this difficulty by appropriate numerical procedure. In the present report, we restrict ourselves to $A \leq 35$, for which reliable calculations are possible.

We have compared our results with earlier calculations for $A=3$ [12], K-harmonic approximation [13], exact diagonalization of the Hamiltonian in one and two dimensions [19] as also with the predictions of the GP equation [8]. As a preliminary calculation we have taken two-body Gaussian interactions of varying range. Our results agree qualitatively with the previous ones, most of which use a contact interaction. This demonstrates the reliability and feasibility of our method. Thus a reliable $a b$ initio calculation for a large but finite number of atoms in a condensate, where individual particles 
interact via realistic two-body interactions, appears feasible. Extension of our method to larger number of particles as also use of more realistic two-body interaction is underway.

\section{Acknowledgements}

This work has been supported by a grant from the Department of Science and Technology (DST), Government of India under a research project. One of the authors (BC) wishes to thank Prof. D. K. Watson for providing a Post-doctoral fellowship at the University of Oklahoma (U.S.A.), where part of the work was done. She also wishes to thank Dr. B. A. McKinney for providing the code for solving the GP equation. 


\section{Appendix}

\section{Hyperspherical variables and hyperspherical harmonics}

\section{A1 : Hyperspherical variables}

The relative motion of the $A=(N+1)$ particle system is described in terms of $N$ Jacobi coordinates defined by eq. (8) and having $3 N$ degrees of freedom. An equivalent set of hyperspherical variables is constituted by the hyperradius $(r)$ defined by eq.(11), $2 N$ spherical polar angles of $\overrightarrow{\zeta_{1}}, \overrightarrow{\zeta_{2}} \ldots ., \overrightarrow{\zeta_{N}}$ and $(N-1)$ hyperangles $\left\{\phi_{2}, \phi_{3}, \ldots . \phi_{N}\right\}$ giving the length of the Jacobi vectors $\overrightarrow{\zeta_{1}}, \overrightarrow{\zeta_{2}} \ldots ., \overrightarrow{\zeta_{N}}$, through

$$
\begin{aligned}
& \zeta_{N}=r \quad \cos \phi_{N} \\
& \zeta_{N-1}=r \sin \phi_{N} \quad \cos \phi_{N-1} \\
& \zeta_{N-2}=r \quad \sin \phi_{N} \quad \sin \phi_{N-1} \quad \cos \phi_{N-2} \\
& \zeta_{2}=r \quad \sin \phi_{N} \quad \sin \phi_{N-1} \ldots \sin \phi_{3} \quad \cos \phi_{2} \\
& \zeta_{1}=r \quad \sin \phi_{N} \quad \sin \phi_{N-1} \ldots \sin \phi_{3} \quad \sin \phi_{2} \\
& \left(\phi_{1}=0\right)
\end{aligned}
$$

Eq. (39) automatically satisfies eq. (11).

\section{A2. Grand orbital operator}

The general grand orbital operator, $L^{2}\left(\Omega_{N}\right)$ of eq. $(15)$ is defined 
through [14]

$$
\begin{aligned}
L_{i}^{2}\left(\Omega_{i}\right)= & \frac{\partial^{2}}{\partial \phi_{i}^{2}}+\left[3(i-2) \cot \phi_{i}+2\left(\cot \phi_{i}-\tan \phi_{i}\right)\right] \frac{\partial}{\partial \phi_{i}}+\frac{l^{2}\left(\omega_{i}\right)}{\cos ^{2} \phi_{i}}+\frac{L_{i-1}^{2}\left(\Omega_{i-1}\right)}{\sin ^{2} \phi_{i}} \\
= & 4\left(1-z_{i}^{2}\right) \frac{\partial^{2}}{\partial z_{i}^{2}}+6\left[2-i\left(1+z_{i}\right)\right] \frac{\partial}{\partial z_{i}}+2 \frac{l^{2}\left(\omega_{i}\right)}{1+z_{i}}+2 \frac{L_{i-1}^{2}\left(\Omega_{i-1}\right)}{1-z_{i}} \\
& (i=2, \ldots \ldots \ldots . . N)
\end{aligned}
$$

where $z_{i}=\cos 2 \phi_{i}, \omega_{i}$ represents the set of two polar angles of $\vec{\zeta}_{i}$ and $\phi_{i}$ 's are given by eq. (39). Note that $L_{1}^{2}\left(\Omega_{1}\right)=l^{2}\left(\omega_{1}\right)$ and $L_{N}^{2}\left(\Omega_{N}\right) \equiv L^{2}\left(\Omega_{N}\right)$ appear in eq. (15).

\section{A3. Hyperspherical harmonics}

An eigenfunction of $L^{2}\left(\Omega_{N}\right)$ is called hyperspherical harmonics $(\mathrm{HH})$ and is given (without angular momentum coupling) by [34]

$$
Y_{[\mathcal{L}]}\left(\Omega_{N}\right)=Y_{l_{1} m_{1}}\left(\omega_{1}\right) \prod_{j=2}^{N} Y_{l_{j} m_{j}}\left(\omega_{j}\right)^{(j)} P_{\mathcal{L}_{j}}^{l_{j}, \mathcal{L}_{j-1}}\left(\phi_{j}\right)
$$

where

$$
\begin{aligned}
{ }^{(j)} P_{\mathcal{L}_{j}}^{l_{j} \mathcal{L}_{j-1}}\left(\phi_{j}\right)= & \left\{\frac{2 \nu_{j} \Gamma\left(\nu_{j}-n_{j}\right) \Gamma\left(n_{j}+1\right)}{\Gamma\left(\nu_{j}-n_{j}-l_{j}-\frac{1}{2}\right) \Gamma\left(n_{j}+l_{j}+\frac{3}{2}\right)}\right\}^{\frac{1}{2}} \\
& \left(\cos \phi_{j}\right)^{l_{j}}\left(\sin \phi_{j}\right)^{\mathcal{L}_{j-1}} P_{n_{j}}^{\nu_{j-1}, l_{j}+\frac{1}{2}}\left(\cos 2 \phi_{j}\right) \quad(j=2,3, \ldots, N)
\end{aligned}
$$

with

$$
\begin{aligned}
\nu_{j}= & \nu_{j-1}+2 n_{j}+l_{j}+\frac{3}{2} \\
= & \mathcal{L}_{j}+\frac{3 j}{2}-1 \\
= & \mathcal{L}_{j-1}+2 n_{j}+l_{j}+\frac{3 j}{2}-1 \\
& \quad(j=2,3, \ldots, N)
\end{aligned}
$$

In eq. (42) $P_{n}^{\alpha, \beta}(x)$ is a Jacobi Polynomial. In eq. (41), $[\mathcal{L}]$ representa the set of quantum numbers $\left\{\left(l_{1}, m_{1}\right),\left(l_{2}, m_{2}\right), \ldots,\left(l_{N}, m_{N}\right), n_{2}, n_{3}, \ldots, n_{N}\right\}$ for a fixed value of grand orbital quantum number $\mathcal{L}=\mathcal{L}_{N}$. The quantum number $\mathcal{L}_{i}$ is defined through

$$
\mathcal{L}_{i}=\mathcal{L}_{i-1}+2 n_{i}+l_{i}
$$


with $\mathcal{L}_{1}=l_{1}$. Hence

$$
\mathcal{L} \equiv \mathcal{L}_{N}=l_{1}+\sum_{j=2}^{N}\left(2 n_{j}+l_{j}\right)
$$

The HH of eq. (41) forms the uncoupled basis. For systems with a good orbital angular momemtum $\vec{L}=\overrightarrow{l_{1}}+\overrightarrow{l_{2}}+\ldots+\overrightarrow{l_{N}}$, one has to couple the individual orbital angular momenta - then the projection quantum numbers $m_{1}, m_{2}, \ldots, m_{N}$ are replaced by the $(N-1)$ intermediately coupled angular momenta and the projection $M$ of $\vec{L}$.

The potential harmonics $(\mathrm{PH})$ given by eq. (17) corresponds to $l_{N}=l$, $l_{1}=l_{2}=l_{3}=\ldots=l_{N-1}=0$, such that $L=l_{N}=l, M=m_{N}=m$ and grand orbital $\mathcal{L}$ $\equiv \mathcal{L}_{N}=2 K+l$ with $n_{2}=n_{3}=\ldots=n_{N-1}=0$ and $K=n_{N}$. Substitution of these in eqs. (41) - (43) gives the PH of eq. (17). 


\section{References}

[1] S. N. Bose, Z. Phys. 26 (1924) 178

[2] A. Einstein, Sitzber. Kgl. Preuss. Akad. Wiss. (1924) 261

[3] A. Einstein, Sitzber. Kgl. Preuss. Akad. Wiss. (1925) 3

[4] K. B. Davis et al, Phys. Rev. 75 (1995) 3969

[5] C. C. Brady et al, Phys. Rev. 75 (1995) 1687

[6] M. H. Anderson et al Science 269 (1995) 198

[7] G. Byam and C. J. Pethick, Phys. Rev. Lett. 76 (1996) 6

[8] F. Dalfovo, S. Giorgini, L. P. Pitaevskii and S. Stingari, Rev. Mod. Phys. 71 (1999) 463

[9] B. D. Esry, Phys. Rev. A55 (1997) 1147

[10] C. J. Pethik and H. Smith, Bose-Einstein Condensation in Dilute Gases (Cambridge University Press, Cambridge, 2001)

[11] A. J. Leggett, Rev. Mod. Phys. 73 (2001) 307

[12] B. D. Esry and C. H. Greene, Phys. Rev. A60 (1999) 1451

[13] J. L. Bohn, B. D. Esry and C. H. Greene, Phys. Rev. A58 (1998) 584

[14] J. L. Ballot and M. Fabre de la Ripelle, Ann. Phys. (N.Y.) 127 (1980) 62

[15] T. K. Das, R. Chattopadhyay and P. K. Mukherjee, Phys. Rev. A50 (1994) 3521

[16] T. K. Das, H. T. Coelho and M. Fabre de la Ripelle, Phys. Rev. C26 (1982) 2281

[17] M. Beiner and M. Fabre de la Ripelle, Lett. Nuovo. Cim. 1 (1971) 584 
[18] T. K. Das and H. T. Coelho, Phys. Rev. C26 (1982) 754

[19] T. Haugset and H. H. Haugerud, Phys. Rev. A57 (1998) 3809

[20] O. Sorensen, D. V. Fedorov, A. S. Jensen and E. Nielsen, Phys. Rev. A65 (2002) 051601(R)

[21] O. Sorensen, D. F. Fedorov and A. S. Jensen, Phys. Rev. A66 (2002) 032507;

O. Sorensen, D. F. Federov and A. S. Jensen, Phys. Rev. Letts. 89 (2002) 173002

[22] M. Fabre de la Ripelle, Few-Body Systems 1 (1986) 181

[23] M. Fabre de la Ripelle, Ann. Phys. (N.Y.) 147 (1983) 281

[24] M. Abramowitz and I. A. Stegun, Handbook of Mathematical Functions, Dover Publications, New York (1972), p.773

[25] T. K. Das and S. Roy, Pramana (Jour. of Phys.) 36 (1991) 305

[26] J. L. Ballot, M. Fabre de la Ripelle and J. S. Levinger, Phys. Rev. C26 (1982) 2301

[27] V. P. Brito, H. T. Coelho and T. K. Das, Phys. Rev. A40 (1989) 3346

[28] R. Chattopadhyay and T. K. Das, Phys. Rev. A56 (1997) 1281

[29] T. K. Das and B. Chakrabarti, to appear in Int. Jour. Mod. Phys. A (2004)

[30] T. K. Das, H. T. Coelho and M. Fabre de la Ripelle Phys. Rev. C26 (1982) 2288

[31] M. A. Khan, T. K. Das and B. Chakrabarti, Int. Jour. Mod. Phy. E10 (2001) 107

[32] B. R. Johnson, J. Chem. Phys. 69 (1978) 4678

[33] I. Elminyaki and J. S. Levinger, J. Chem. Phys. 82 (1985) 905 
[34] F. Zernike and H. C. Brinkman, Proc. Kon. Ned. Acad. Wensch 33 (1935) 3 
Table 1. Calculated ground state energy per particle (in o.u.) of the condensate containing $A$ particles for various $K_{\max }$ values, showing convergence trend as $K_{\max }$ increases $\left(V_{0}=3.1985 \times 10^{6}\right.$ o.u. and $r_{0}=0.005$ o.u. $)$

\begin{tabular}{|l|l|l|l|l|l|l|}
\hline $\begin{array}{l}K_{\max } \\
A\end{array}$ & 2 & 4 & 6 & 8 & 10 & 12 \\
\hline 3 & 1.50041 & 1.50031 & 1.50026 & 1.50023 & 1.50021 & 1.50019 \\
5 & 1.50123 & 1.50117 & 1.50112 & 1.50108 & 1.50104 & 1.50101 \\
10 & 1.50350 & 1.50348 & 1.50346 & 1.50344 & 1.50342 & 1.50340 \\
15 & 1.50453 & 1.50451 & 1.50450 & 1.50449 & 1.50449 & \\
20 & 1.50539 & 1.50538 & 1.50537 & 1.50536 & 1.50536 & \\
25 & 1.50618 & 1.50617 & 1.50617 & 1.50616 & 1.50616 & \\
30 & 1.50693 & 1.50692 & 1.50692 & 1.50691 & & \\
35 & 1.50764 & 1.50763 & 1.50763 & 1.50766 & & \\
\hline
\end{tabular}

Table 2. Calculated excitation energies (in o.u.) of the first three excited states for different numbers $(A)$ of ${ }^{87} R b$ atoms (parameters as in Table. 1).

\begin{tabular}{|l|l|l|l|}
\hline$A$ & $1^{\text {st }}$ & $2^{\text {nd }}$ & $3^{\text {rd }}$ \\
\hline 3 & 2.00116 & 4.00283 & 6.00494 \\
5 & 2.00130 & 4.00428 & 6.00962 \\
10 & 2.00231 & 4.00705 & 6.01268 \\
15 & 2.00355 & 4.0130 & 6.03147 \\
20 & 2.00471 & 4.01647 & 6.04604 \\
25 & 2.00671 & 4.03225 & 6.12762 \\
30 & 2.03276 & 4.08846 & 6.17127 \\
35 & 2.08319 & 4.10225 & 6.27118 \\
\hline
\end{tabular}




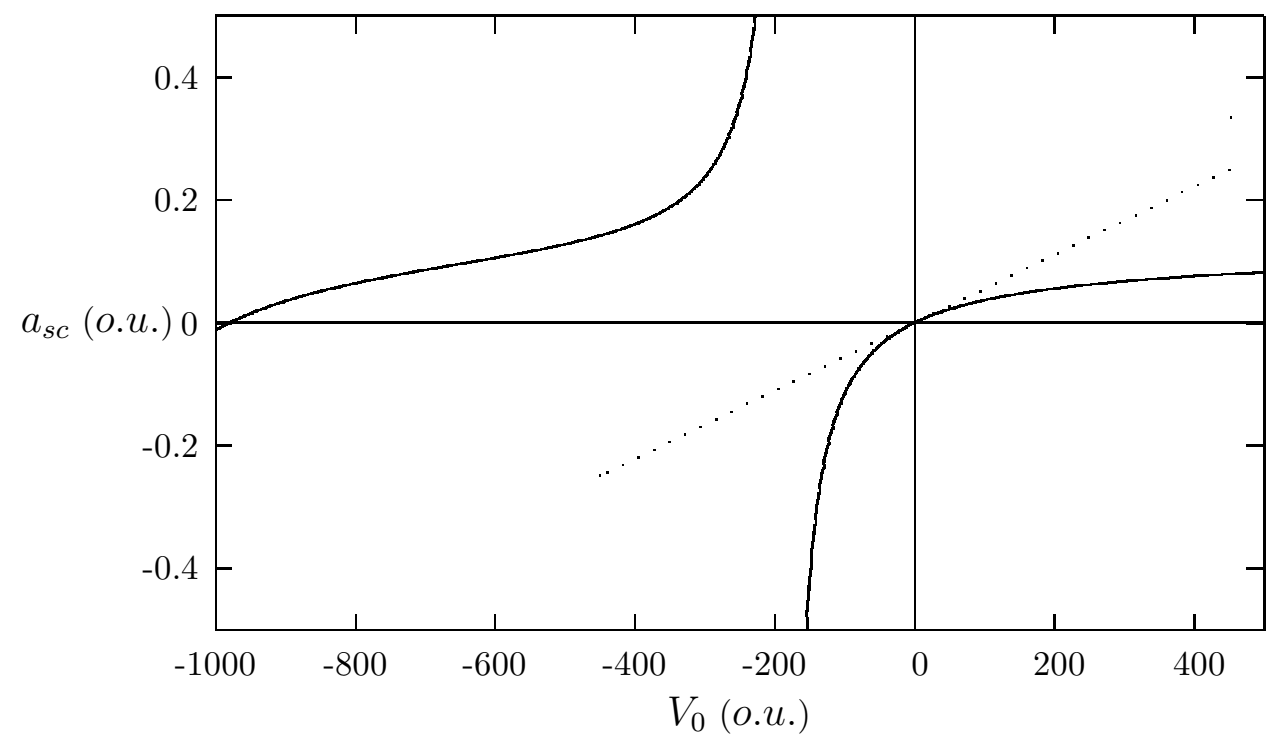

Fig. 1 - Plot of calculated $a_{s c}$ as a function of $V_{0}$ for $r_{0}=0.0855$ o.u.. The dotted line corresponds to the Born approximation $\left(a_{s c}^{(B)}\right)$. 


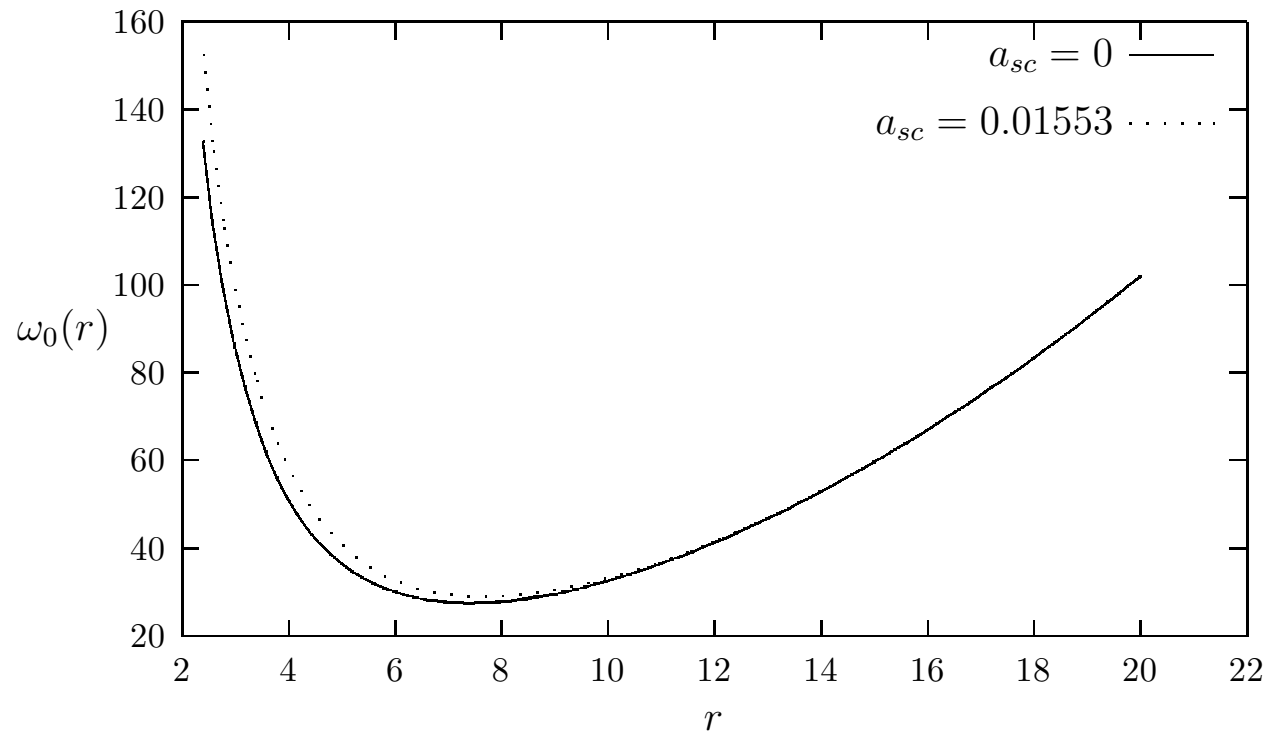

Fig. 2 - Lowest eigen potential for $A=20$ as a function of $r$. Continuous curve is for $a_{s c}=0$ (no two-body interaction) and the dotted curve is for a repulsive interaction $\left(a_{s c}=0.01553\right.$ о.и.). 


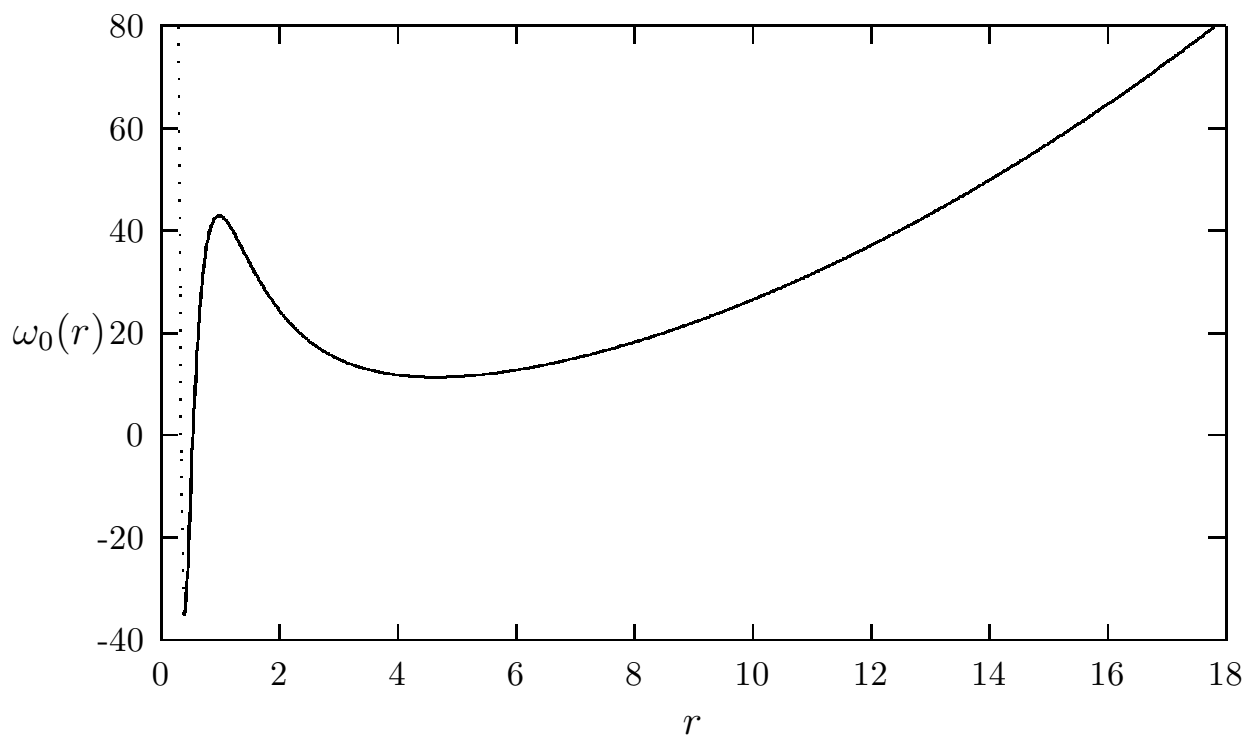

Fig. 3 - Plot of $\omega_{0}(r)$ as a function of $r$ (dotted and continuous curves together) for $A=10$ (subcritical number) for a model attractive two-body interaction $\left(V_{0}=-100\right.$ o.u., $r_{0}=$ 0.0855 o.u.), which corresponds to $a_{s c}=-0.1176$ o.u. 


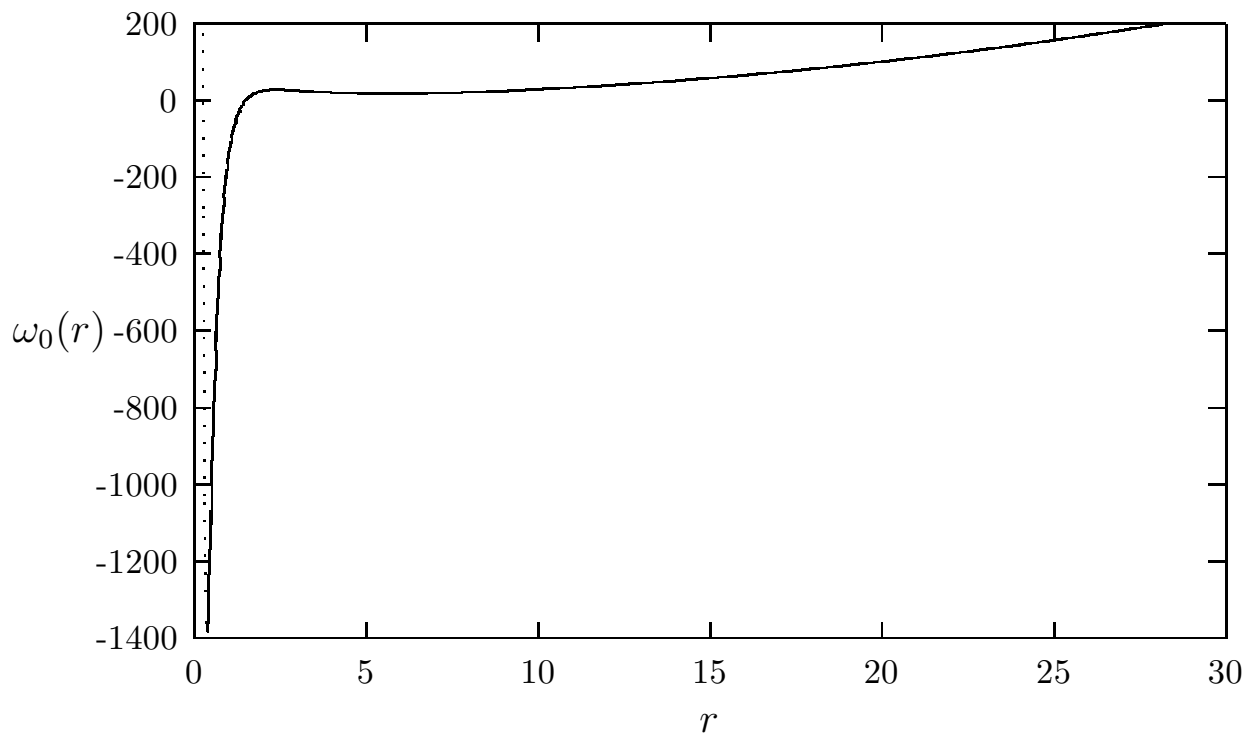

Fig. 4 - Plot of $\omega_{0}(r)$ as a function of $r$ (dotted and continuous curves together) for $A=16$ (critical number) for the same attractive two-body potential as in Fig. 3. 


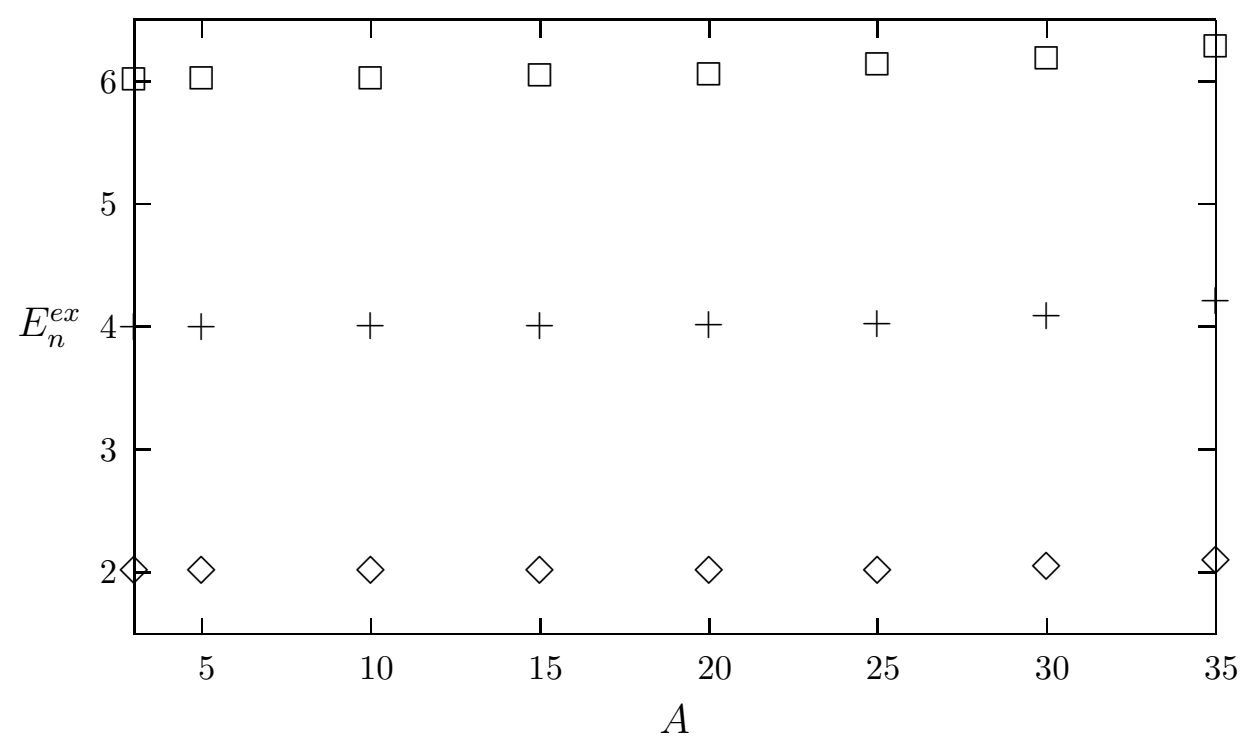

Fig. 5 - Three low-lying excitation frequencies for various values of particle number $(A)$, corresponding to the JILA experiment with ${ }^{87} R b$ atoms ( $a_{s c}=100 \mathrm{Bohr}$, oscillator frequency $=200 H z)$. Energies are in oscillator units.

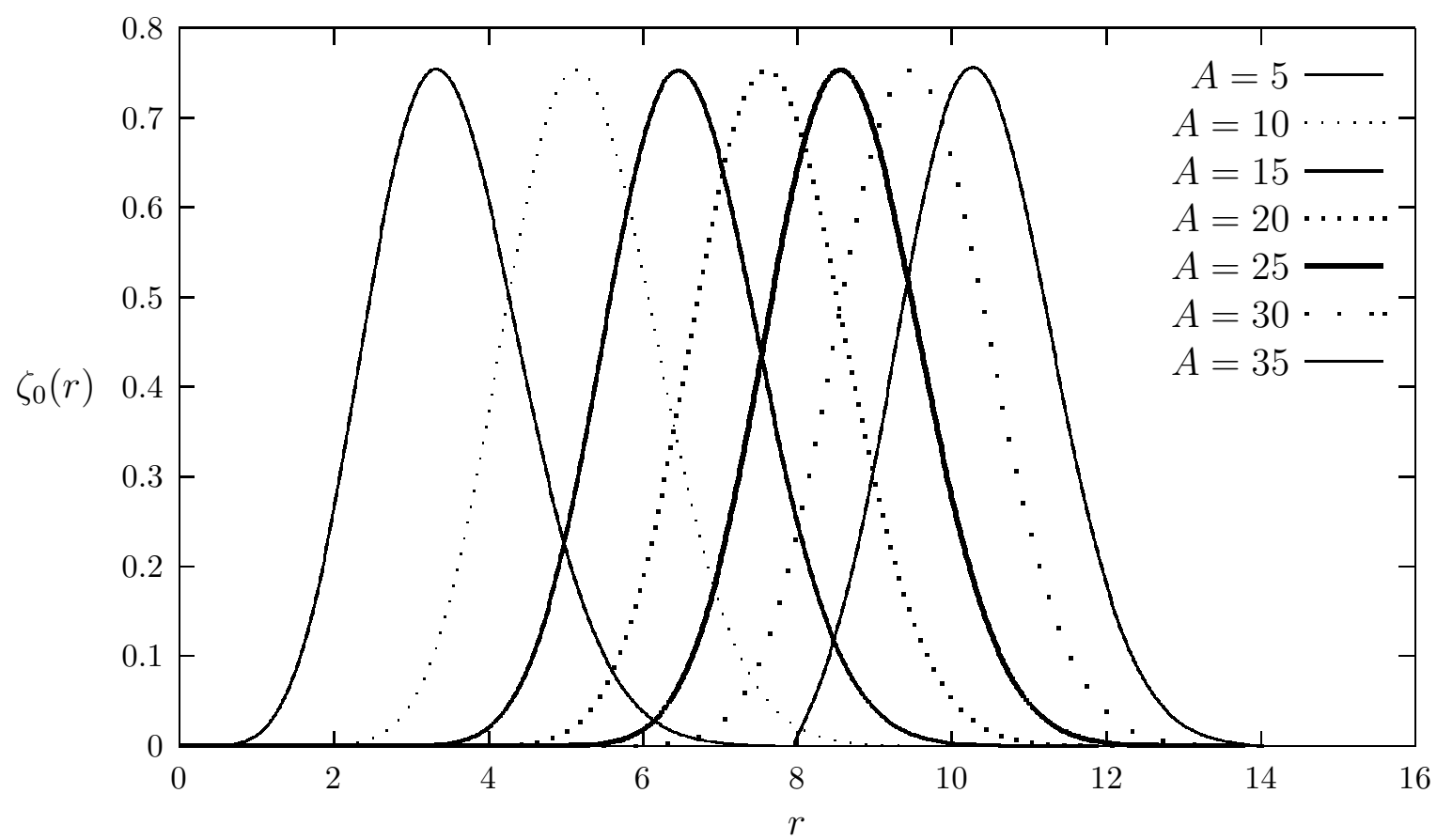

Fig. 6 - Plot of ground state wave function as a function of hyperradius $(r)$ for various indicated value of $A$, in the chosen trap. 


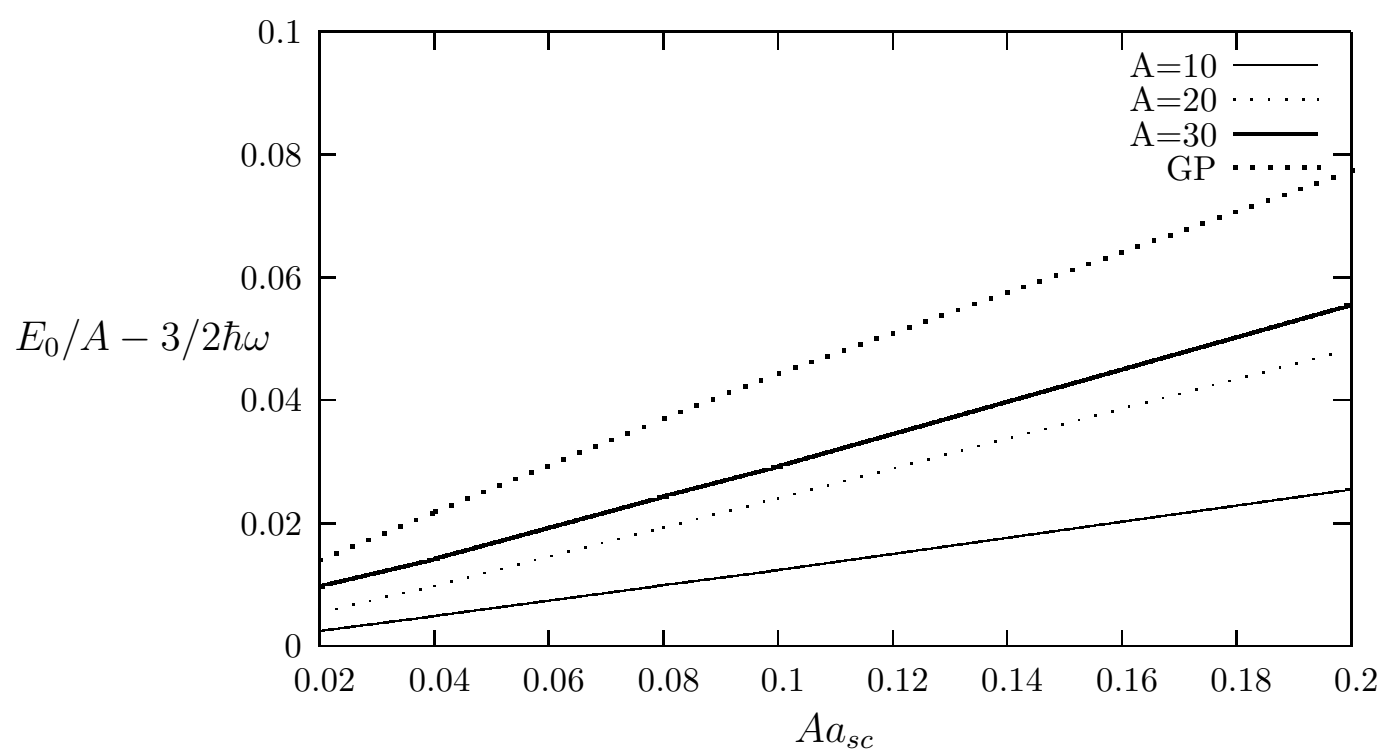

Fig. 7 - Plot of ground state energy per particle $\left(E_{0} / A-3 / 2 \hbar \omega\right)$ as a function of $A a_{s c}$ for a repulsive interaction for indicated values of $A$ and the GP results. 